\title{
Building A Portable Computer Lab With BOOTABLE USB HARD DRIVES USING VIRTUALISATION
}

\author{
Hao Shi \\ School of Engineering and Science, Victoria University, Melbourne, Australia \\ hao.shi@vu.edu.au
}

\begin{abstract}
Centralization of services is a global trend in universities worldwide. IT services in a university become a solo department to provide common IT support for the entire university community such as staff, students and visitors. Special services required for Computer Science and Information Technology courses are quite often not part of IT service agreements. The shared common computer labs are tightly controlled by IT services and could not be used to deliver some specialised course such as Microsoft Active Directory Configuration and Management. A dedicated computer lab is no longer a viable option to provide a practical solution with three-year University leased computers. The challenge in providing a long-term solution heavily relies on the individual academic staffs who deliver such courses. There is an urgent need to find a new long-term working solution for this problem. After numerous search and research in finding an optimal solution, a portable computer lab using virtualisation is proposed. Instead of using a desktop computer in a common lab, each student enrolled in the course such as Microsoft Active Directory is allocated a customised USB hard dive. The USB drive contains a bootable Windows 7 Operating System as well as six Windows Servers operated under Microsoft Windows Virtual PC. This not only allows the students to use a common computer lab but also enable them to totally control their learning with flexibility which was not achievable in the past. This paper aims to share the knowledge as well as tricks and tips gained through the process and to provide step by step instructions for building the customised USB hard dive. It is expected that the concept of a 'portable' computer lab using virtualisation will provide insight into delivery of IT courses in the future.
\end{abstract}

\section{KEYWORDS}

Portable Computer Lab, Virtualisation, Bootable USB Hard Drive and IT courses

\section{INTRODUCTION}

Centralization of activities/functionalities is a global trend in a business world [1]. Australian universities are catching up with the business world with many services offered at the universities being centralised. IT services are a typical example, which used to exist at the individual School/Department who provided special needs to IT courses. They now only have a budget to support computer software installation, maintenance and management. With a three-year lease agreement with university computers, the computer hardware supports become a phone call to fetch the suppliers for parts replacement. Speciality services are vanishing and the skill sets required at IT service is also diminishing in much faster paces than ever. Academic staffs, who deliver such speciality courses, constantly face challenges to find a practical solution quickly without IT support, sometimes without budget or support. David Marshall stated in his InfoWorld article "Virtualization allows you to easily build out a self-contained lab or test environment,

DOI : $10.5121 /$ ijcsit.2012.4504 
operating on its own isolated network."[2]. Even though IT industry has virtualisation technologies in its dedicated computer labs to deliver industry courses such as Microsoft and Cisco certificates, the information is not publicly available. Academic staffs are distracted from their major focus on teaching and research to quickly identify a possible solution, for this instance, virtualisation [3] to put it into use quickly. In this paper, a portable computer lab using virtualisation is proposed. The detail of building a customised bootable USB hard dive using Window Virtual PC is full documented. Software and Hardware requisites are described in Section 2. Building a bootable Windows 7 USB Hard Drive is detailed in Section 3. Installation of Virtual Network is presented in Section 4. Conclusion and future works are given in Section 5.

\section{Software And Hardware Prerequisites}

The following downloadable toolkits are the prerequisite:

- A Laptop or Desktop Computer with Windows 7 OS installed

- Microsoft@ Hardware-Assisted Virtualization Detection Tool (Havdtectiontool.exe)

Downloadable from http://www.microsoft.com/en-us/download/details.aspx?id=592

This software allow user to check if a computer supports virtualisation [4].

- SetupImgBurn (free license)

Downloadable from http://www.imgburn.com/

A lightweight CD / DVD / HD DVD / Blu-ray burning application is used to create image files [5].

- DAEMON Tools Lite (free license)

Downloadable from http://www.daemon-tools.cc/eng/products/dtLite

A software is used to mount a disk image such as .iso file [6].

\section{Building A Bootable Windows 7 USB HaRd Drive}

There are hundreds of websites which provide detail information about how to create a bootable Windows 7 USB flash drive, unfortunately not hard drive. Although Google does provide good sources for a solution, it still requires enormous efforts in online search and research to evaluate each possible option. In the end, an online tutorial 'How to install Windows Vista/7/SVR2K8 onto a USB drive' is found from RMPreUSB.com [7]. After a few times of 'trial and error', a bootable Windows 7 hard drive is created. However, it should be pointed out that it is only working with a beta version of Windows 7 because the license version of Windows 7 for some unknown reasons failed to work even though much testing was conducted without being able to identify the real cause. For this particular reason, the detail is provided in the following subsection.

\subsection{Software and Hardware Requirements}

The software and hardware listed below are required to create a bootable Windows 7 USB Hard Drive

- A USB hard Drive with minimum 128GB capacity 10 GB is enough for Windows 7 OS but since six Windows Server 2008 virtual machines needs to be added to the bootable drive, a high capacity USB hard drive is a better choice.

- NT6_Fast_Installer.zip Downloadable from http://www.rmprepusb.com/documents/rmprepusb-beta-versions 
International Journal of Computer Science \& Information Technology (IJCSIT) Vol 4, No 5, October 2012

- Microsoft Windows ${ }^{\circledR}$ Automated Installation Kit (WAIK) Supplement for Windows ${ }^{\circledR} 7$ SP1 (KB3AIK_EN.iso)

Downloadable from http://www.microsoft.com/en-au/download/details.aspx?id=5188

- 64-bit Windows 7 SP1 (X17-24281.iso)

Downloadable from http://techdows.com/2011/07/download-windows-7-integrated-withsp1-iso-official-direct-download-links.html

The temporary reference key is H72RH-R3437-T2WRB-GJBFQ-28JP6.

\subsection{Installation of Windows 7 SP1 onto a USB Hard Drive}

First step: install Microsoft WAIK on the current laptop or desktop computer by choosing run.exe from 'AutoPlay' pop-up window. Restart computer when finished, and check that bootsect.exe, bcdboot.exe (already in Win7) and imagex.exe are installed properly.

Second step: unzip NT6_Fast_Installer.zip to a folder like NT6 [7].

Third step: mount the image file X17-24281.iso using DAEMON Tools Lite by simply double clicking .iso file and record the Drive letter such as E:I.

Fourth step: open the NT6 folder, right-click installer.exe and 'Run as Administrator'. Follow the instructions on screen by providing $\mathrm{E}$ as a drive Letter (or from $3^{\text {rd }}$ Step for an answer) as shown in Figure 1(a). Now select the Install.wim file from Windows 7 mounted ISO volume as shown in Figure 1(b). Choose Windows 7 Professional edition as shown in Figure 1(c). Once installation is successfully completed, restart computer.

The video instructions and screen snapshots can be found at RMPreUSB.com [7].

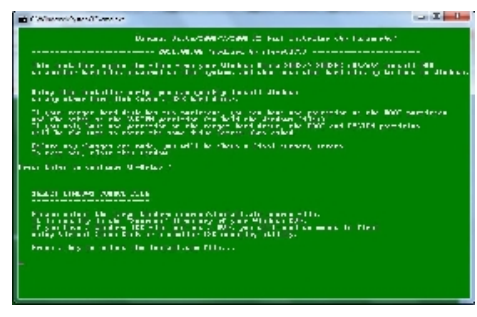

(a)

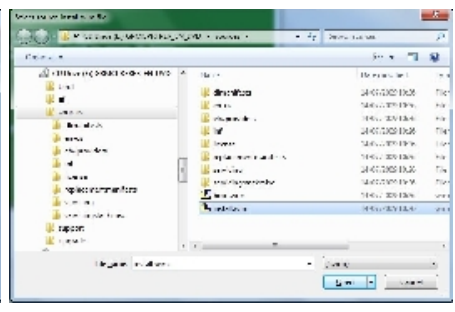

(b)

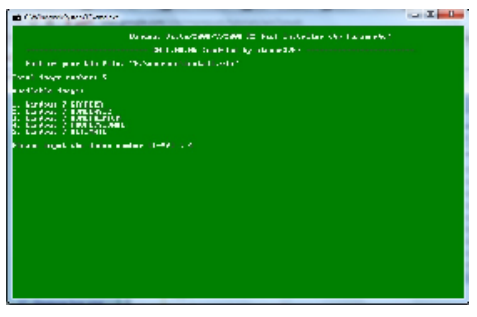

(c)

Figure 1. NT6 Fast Installer Screens [7]

\subsection{Running Windows 7 SP1 from the bootable USB Hard Drive}

During the restart, press F12 or F8 or F2 which are common keys for configuring BISO to change Boot or Hard Disk orders. Make sure that the bootable USB hard disk is on top of the list. Then Press F10 with confirmation of "Yes" to accept the change. Then Windows 7 will automatically run from the attached USB HD.

Adjust 'Display' and 'Time Zone' if necessary and create a login such as [8]:

Username: ADS

Password: MSPress\#1 


\section{InStALLATION OF VIRTUAL NETWORK}

\subsection{Software Requirements}

A number of software tool are required to create a virtual network on the bootable USB drive:

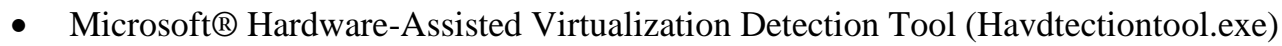
Downloadable from http://www.microsoft.com/en-us/download/details.aspx?id=592

- Windows Virtual PC (Windows6.1-KB958559-x64-RefreshPkg)

Downloadable from http://www.microsoft.com/windows/virtual-pc/

- 32-bit Windows Server 2008 (KRMSFRE_EN_DVD.iso)

Downloadable from http://www.microsoft.com/en-us/download/details.aspx?id=8371

\subsection{Installation of Windows Virtual PC (WVPC)}

It's time to run havdtectiontool.exe and ensure that the laptop or desktop computer supports or switch on virtualisation. Then Windows Virtual PC can be installed. This is a straightforward process. Double-click on Windows6.1-KB958559-x64-RefreshPkg and follow the instruction on the screen. Restart the computer when completed. Please note that Windows Virtual PC only supports 32-bit Operating System so that Windows Server 2008 R2 (64-bit) cannot be installed under WVPC. It should be pointed out that VMware is regarded as a leader in virtualisation [9, 10] and may provide a better option but it has to be left for the next update due to time constraint. For example, VMware can replace Windows Virtual PC to support 64-bit Windows Server 2008 R2 or the latest Windows Server 2012.

\subsection{Installation of Windows Server 2008 Enterprise on WVPC}

Run WVPC from the shortcut on desktop or Start => All Programs => Windows Virtual PC => Windows Virtual PC. Now it is ready to install Windows Servers 2008 under WVPC, which have built-in Active Directory components. The portable Active Directory Lab consists of six virtual machines, namely, two RWDC (Writeable Domain Controller), two RODC (Read-Only Domain Controller) and two SCDC (Sever Core Domain Controller) [8].

\subsubsection{Installation of RWDC virtual server machines on WVPC}

The following are stepped instructions for RWDCxx where $\mathrm{xx}$ represents a virtual machine number so that two students in a team have unique odd and even numbers for their machines:

1) Under menubar, click on 'Create virtual machine' with the following setting:

Name: RWDCxx

Location: E:IECB3142VM (or drive letter from $3^{\text {rd }}$ Step in Section 3.2)

RAM: $800 \mathrm{MB}$

Hard Disk: 80GB under special setting

Keep all default settings

2) Select RWDCxx Virtual Machine and Click on Settings (under menubar)

3) Select on DVD Drive and tick 'Open an ISO image'. Browse Desktop/EBC3145files folder and select KRMSFRE_EN_DVD.iso (32-bit Windows Server 2008 image file)

4) Click on Networking with the following settings:

Number of Networking adapters: 1 or 2 (WiFi Internet Connection)

Adapter 1: Internal Network

Adapter 2 (optional):Atheros AR9285 Wireless Network Adapter 
Click 'OK' to finish settings.

Students may be confused at the beginning with two adaptors. It is wise just choose one Adapter and add the second one if needed.

5) Double-click on RWDCxx to install Windows Server 2008. Alt-Tab allows to move cursor between the Windows 7 OS and the Virtual Machine RWDCxx.

5.1) Choose English (Australia) for Time and currency format, then Next

5.2) Un-tick 'Automatically activate Windows when I am online' then Next

5.3) 'Do you want to enter product key?' No

5.4) Select 'Windows Server 2008 Enterprise (Full Installation)'

Tick 'I have selected the edition of Windows that I purchased, then Next

5.5) Tick 'I accept the license terms, then Next

5.6) Click on 'Custom (advanced)'

5.7) Click Next on 'Disk 0 Unallocated Space'

5.8) Install Windows start ...

5.9) Set Administrator Password: e.g. MSPress\#1, then click on 'Arrow'

5.10) 'Password has been changed', Click 'OK'

5.11) Installation is completed.

6) Install Integration Component which is an extra step for user to move between Windows 7 OS and Virtual Machine (VM) seamless.

6.1) Click on Tools from menubar

6.2) Select Install Integration Component, Click 'Continue'

6.3) If no response setup, RETSTART computer (don't look for DVD Drive just yet)

6.4) Once computer restart, Click Start $=>$ Computer, then Click on DVD Drive D:

6.5) Run setup to Install Virtual PC integration Component

6.6) Click 'Finish' and restart computer

6.7) Click 'Tools' from menubar and Select 'Enable Integration Component'

6.8) Click 'Other user', for example

Username: Administrator

Password: MSPress\#1

Tick 'Remember my credienticals'

NOW GUI is integrated and the cursor can be freely moved around between OS and VM.

7) Customize RWDCxx with the following configuration parameters

7.1) Set time zone: GMT+10 for Melbourne

7.2) Configure Networking: Local Area Connection =>ipv4 properties

IP: 192.168.1.11 (as a sample)

Subnet Mask: 255.255.255.0

Default Gateway: 192.168.1.1

DNS Server Address IP: 192.168.1.1

7.3) Provide computer name and domain

Computer Description: Writeable Domain Controller

Computer Name: RWDCxx

7.4) Restart Computer for changes

ALWAYS shut down the bootable USB hard drive so that it can be used on a different laptop or desktop computer to avoid hardware compatibility issues when re-boot next time. 


\subsubsection{Installation of other five Virtual Machines on WVPC}

Repeat the Steps 1) to 7) in Section 4.3.1 to build three more Windows Server 2008 virtual machines with the following specifications:

$$
\begin{aligned}
\text { Step 1) Name: RWDCyy } \\
\text { IP: 192.168.1.12 } \\
\text { Step 1) Name: RODCxx } \\
\text { IP: 192.168.1.13 } \\
\text { Step 1) Name: RODCyy } \\
\text { IP: } 192.168 .1 .14
\end{aligned}
$$

Repeat the Steps 1) to 7) in Section 4.3.1 to build two more Windows Server 2008 Core virtual machines with the following modifcation:

$$
\begin{aligned}
\text { Step 1) Name: SCDCxx } \\
\text { IP: 192.168.1.15 } \\
\text { Step 1) Name: SCDCyy } \\
\text { IP: 192.168.1.16 }
\end{aligned}
$$

Step 5.4) Select 'Windows Server 2008 Enterprise (Server Core Installation)'

Now six virtual machines (RWDCxx, RWDCyy, RODCxx, RODCyy, SCDCxx and SCDCyy) are installed on the bootable USB Hard Drive as shown in Figure 2.

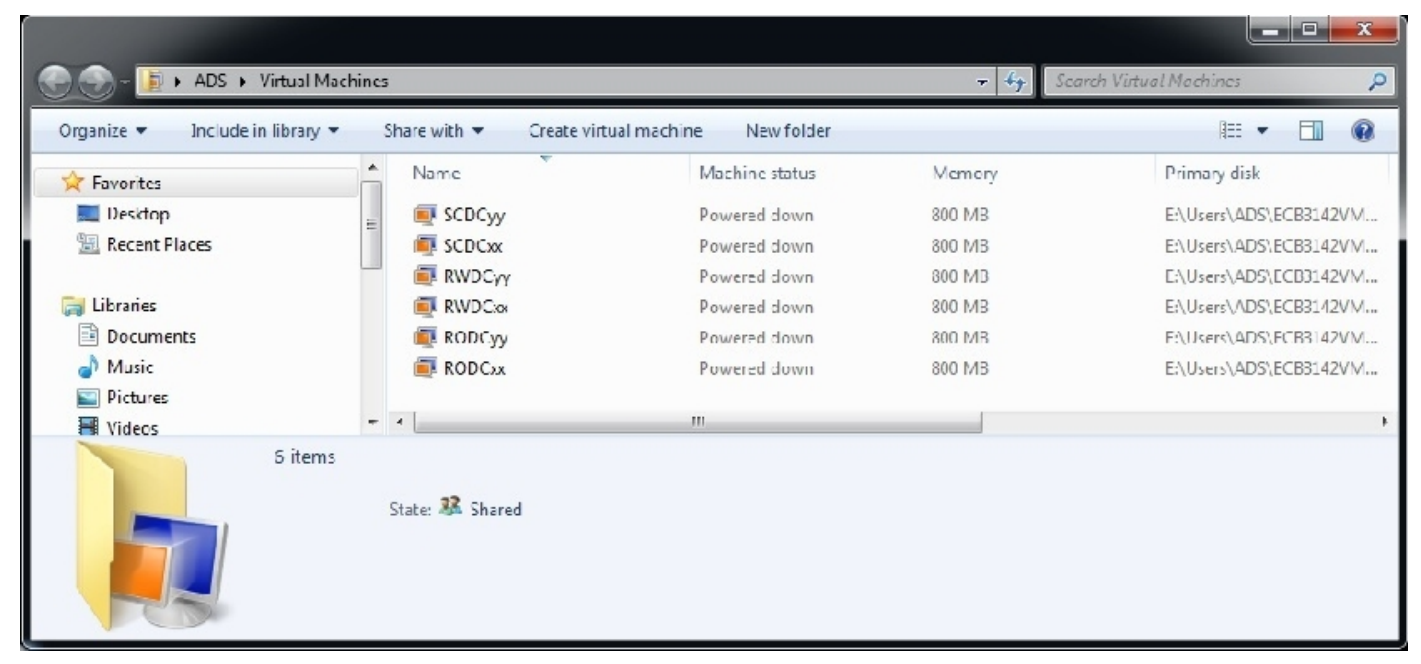

Figure 2. Windows Virtual PC running six 32-bit Windows Server 2008

\subsection{Virtual Network and USB Clone}

Theoretically the virtual network is established if all the required virtual machines are running under WVPC. However, due to the firewall or security setting, quite often the six virtual machines won't be able to communicate with one another. Ping, a computer network administration utility can be employed to test the reachability of a host on an Internet Protocol (IP) network [11]. For example, under Command Prompt, RWDCxx machine can ping RODCyy machine as shown in Figure 3 (a). 
If ping doesn't work as shown in Figure 3(b), make sure that "file sharing" is switched on. If the virtual machines need to access the Internet, the $2^{\text {nd }}$ Adapter such as WiFi network card need to be configured to connect to a WiFi Access Point.

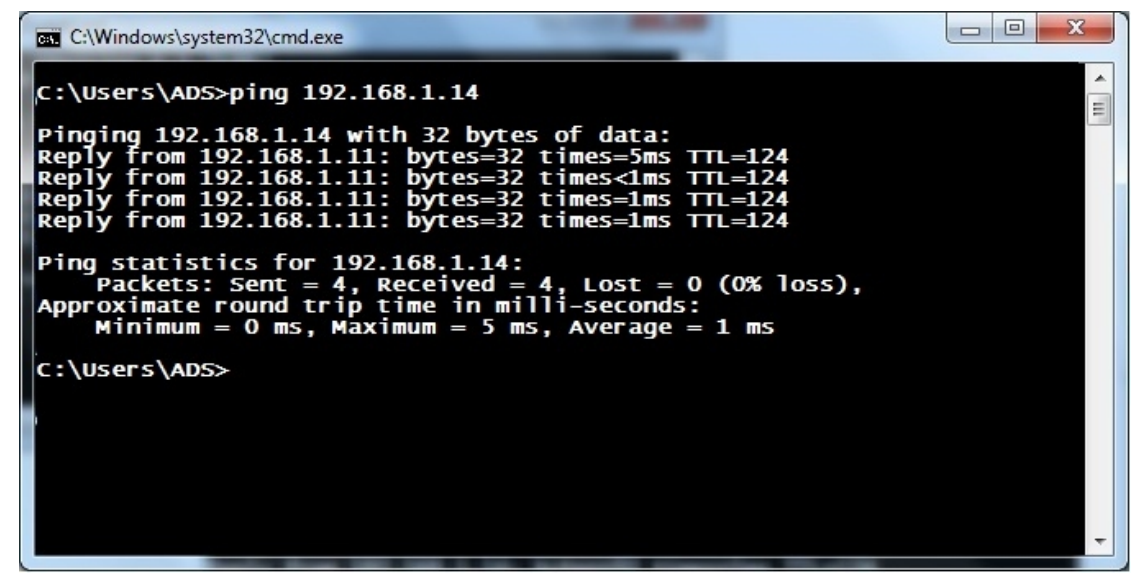

(a) ping is working and return the round-trip time

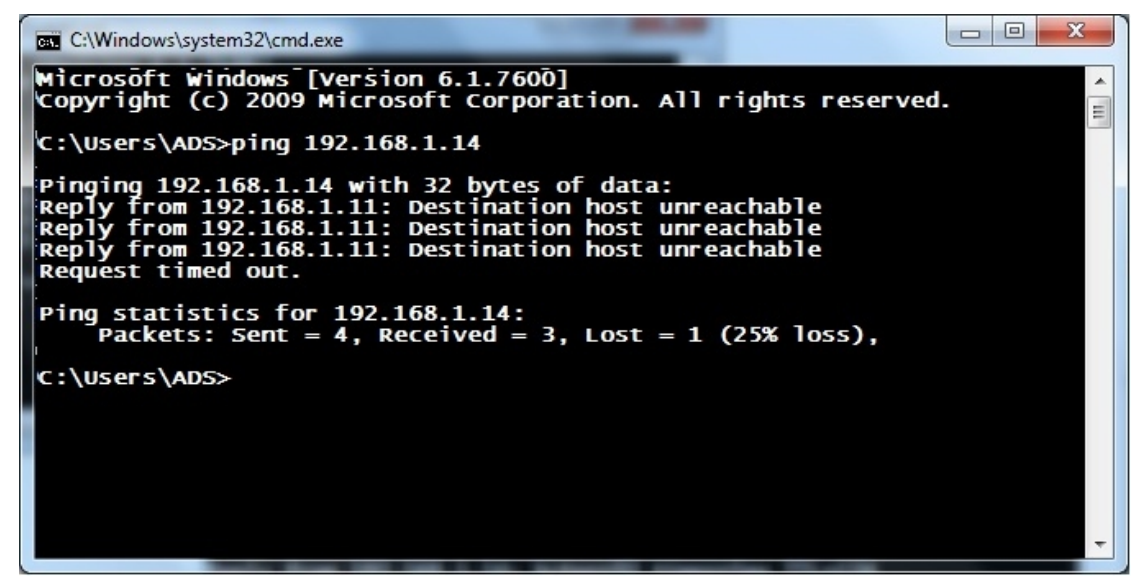

(b) ping doesn't work

Figure 3. Command Prompt Window

Once the bootable USB Hard Drive and six virtual machines are fully tested, the USB can be cloned for distribution to students. Because the bootable USB contains the Windows 7 OS, it cannot be simply copied and special utility software such as SeagateDiskWizard.exe is needed to clone the USB Drive. Since the bootable USB contains over 100 GB contents, it takes more than 180 minutes to clone via USB2.0 or 20 minutes via USB 3.0. It's be better to purchase an USB clone docking for a class size over 100 to have a quick turnover for any last minute update.

\section{CONCLUSION AND FUTURE WORK}

A bootable USB Widows 7 Hard Drive containing six virtual Windows Server 2008 servers is fully implemented. First a bootable USB Hard Drive is created using NT6 and Windows 7 SP1 beta version. Then the virtualisation is established using Microsoft Windows Virtual PC. Finally six Windows Server 2008 virtual machines are successfully installed under the virtualisation 
environment. This portable solution not only allows the students to totally control their learning but also provides a new thought for academic staff in delivering speciality IT courses in the future. It is unprecedented that IT students do not need to queue outside computer labs for their turns to conduct their labs and they can fully control their learning pace and locations. The concept of a portable computer lab demonstrated in the paper could be further improved using VMware which not only support multiple platforms but also 64-bit operating system. In conclusion, the results are very convincing and could provide a practical solution for future IT courses even though the search and research may take a lot of efforts.

\section{REFERENCES}

[1] Procurement Solutions from SAP, (02011 SAP AG. http://www.sap.com/lines-of-business/procurement/solutions-overview.epx

[2] David Marshall, Top 10 benefits of server virtualization, 2 November 2011, http://www.infoworld.com/d/virtualization/top-10-benefits-server-virtualization-177828

[3] Xiaofei Liao, Xiao Xie, Hai Jin, "Sharing Virtual USB Device in Virtualized Desktop", 2011 Fourth International Symposium on Parallel Architectures, Algorithms and Programming (PAAP), 9-11 Dec. 2011, Tianjin, China, pp. $156-160$.

[4] Microsoft ${ }^{\circledR}$ Hardware-Assisted Virtualization Detection Tool http://www.microsoft.com/enus/download/details.aspx?id=592

[5] SetupImgBurn (free license) http://www.imgburn.com/

[6] DAEMIN Tools Lite (free license) http://www.daemon-tools.cc/eng/products/dtLite

[7] How to install Windows Vista/7/SVR2K8 onto a USB drive, RMPreUSB.com, http://www.rmprepusb.com/tutorials/win7onusb

[8] Exam 70-640 Microsoft Office Academic Course, Windows Server 2008 Active Directory Configuration - Lab Manual, MCTS Exam 70-640, (c) 2009 Wiley.

[9] Hardware virtualization, http://en.wikipedia.org/wiki/Platform_virtualization

[10] VMware, http://www.vmware.com/virtualization/

[11] Ping, http://en.wikipedia.org/wiki/Ping_(networking_utility)

\section{Author}

Dr. Hao Shi is an Associate Professor in School of Engineering and at Victoria University, Melbourne, Australia. She completed her $\mathrm{PhD}$ in the area of Computer Engineering at University of Wollongong and obtained her Bachelor of Engineering degree at Shanghai Jiao Tong University, China. She has been actively engaged in R\&D and external consultancy activities. Her research interests include p2p Network, Location-Based Services, Web Services, Computer/Robotics Vision, Visual Communications, Internet and Multimedia Technologies.

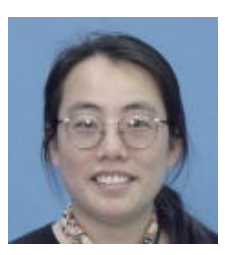

